\title{
WAVELET MULTIPLIERS AND SIGNALS
}

\author{
Z. $\mathrm{HE}^{\mathrm{l}}$ and M. W. WONG ${ }^{1}$
}

(Received 26 March 1996; revised 6 August 1996)

\begin{abstract}
The Schatten-von Neumann property of a pseudo-differential operator is established by showing that the pseudo-differential operator is a multiplier defined by means of an admissible wavelet associated to a unitary representation of the additive group $\mathbb{R}^{n}$ on the $C^{*}$-algebra of all bounded linear operators from $L^{2}\left(\mathbb{R}^{n}\right)$ into $L^{2}\left(\mathbb{R}^{n}\right)$. A bounded linear operator on $L^{2}(\mathbb{R})$ arising in the Landau, Pollak and Slepian model in signal analysis is shown to be a wavelet multiplier studied in this paper.
\end{abstract}

\section{Introduction}

Let $\sigma \in L^{\infty}\left(\mathbb{R}^{n}\right)$. Then we define the linear operator $T_{\sigma}: L^{2}\left(\mathbb{R}^{n}\right) \rightarrow L^{2}\left(\mathbb{R}^{n}\right)$ by

$$
T_{\sigma} u=\mathscr{F}^{-1} \sigma \mathscr{F} u, \quad u \in L^{2}\left(\mathbb{R}^{n}\right),
$$

where $\mathscr{F}$ and $\mathscr{F}^{-1}$ are the Fourier transformation and inverse Fourier transformation respectively. Throughout the paper, the Fourier transform $\mathscr{F} u$, sometimes denoted by $\hat{u}$, of a function $u$ in $L^{2}\left(\mathbb{R}^{n}\right)$, is defined by

$$
\mathscr{F} u=\lim _{R \rightarrow \infty}\left(\chi_{R} u\right)^{\widehat{\gamma}}
$$

where $\chi_{R}$ is the characteristic function of the ball with centre at the origin and radius $R$,

$$
\left.\left.\left(\chi_{R} u\right)\right\urcorner \xi\right)=(2 \pi)^{-n / 2} \int_{\mathbb{R}^{n}} e^{-i x \cdot \xi} \chi_{R}(x) u(x) d x, \quad \xi \in \mathbb{R}^{n}
$$

and the convergence of $\left(\chi_{R} u\right)^{-}$to $\mathscr{F} u$ is understood to be in $L^{2}\left(\mathbb{R}^{n}\right)$. It is well-known that $T_{\sigma}: L^{2}\left(\mathbb{R}^{n}\right) \rightarrow L^{2}\left(\mathbb{R}^{n}\right)$ is a bounded linear operator.

\footnotetext{
${ }^{1}$ Department of Mathematics and Statistics, York University, 4700 Keele Street, North York, Ontario M3J 1P3, Canada.

(C) Australian Mathematical Society 1999, Serial-fee code 0334-2700/99
} 
Let $\phi$ be any function in $L^{2}\left(\mathbb{R}^{n}\right) \cap L^{\infty}\left(\mathbb{R}^{n}\right)$ such that $\|\phi\|_{2}=1$, where \|\|$_{2}$ is the norm in $L^{2}\left(\mathbb{R}^{n}\right)$. The aim of this paper is to make precise the definition of the pseudo-differential operator $\phi T_{\sigma} \bar{\phi}: L^{2}\left(\mathbb{R}^{n}\right) \rightarrow L^{2}\left(\mathbb{R}^{n}\right)$, where $\sigma$ is a function in $L^{p}\left(\mathbb{R}^{n}\right), 1 \leq p \leq \infty$, and to prove that the resulting bounded linear operator is in the Schatten-von Neumann class $S_{p}$. To this end, we first prove in Section 2 that if $\sigma \in L^{\infty}\left(\mathbb{R}^{n}\right)$, then the pseudo-differential operator $\phi T_{\sigma} \bar{\phi}: L^{2}\left(\mathbb{R}^{n}\right) \rightarrow L^{2}\left(\mathbb{R}^{n}\right)$ can be realized as a wavelet multiplier associated to a unitary representation $\pi$ : $\mathbb{R}^{n} \rightarrow B\left(L^{2}\left(\mathbb{R}^{n}\right)\right)$ of the additive group $\mathbb{R}^{n}$ on the $C^{*}$-algebra of all bounded linear operators from $L^{2}\left(\mathbb{R}^{n}\right)$ into $L^{2}\left(\mathbb{R}^{n}\right)$. This connection explains the impetus for the study of the pseudo-differential operator $\phi T_{\sigma} \bar{\phi}: L^{2}\left(\mathbb{R}^{n}\right) \rightarrow L^{2}\left(\mathbb{R}^{n}\right)$ and also reveals that the technique in the paper [3] by He and Wong can be exploited in this paper. We show how this can be done in Sections 3 and 4. In Section 5, we show that, by choosing the admissible wavelet $\phi$ and the symbol $\sigma$ appropriately, the wavelet multiplier $\phi T_{\sigma} \bar{\phi}: L^{2}(\mathbb{R}) \rightarrow L^{2}(\mathbb{R})$ is unitarily equivalent to a scalar multiple of a very basic bounded linear operator on $L^{2}(\mathbb{R})$ arising in the Landau, Pollak and Slepian model in signal analysis.

\section{The wavelet connection}

Let $\pi: \mathbb{R}^{n} \rightarrow B\left(L^{2}\left(\mathbb{R}^{n}\right)\right)$ be the unitary representation of the additive group $\mathbb{R}^{n}$ on the $C^{*}$-algebra $B\left(L^{2}\left(\mathbb{R}^{n}\right)\right)$ of all bounded linear operators from $L^{2}\left(\mathbb{R}^{n}\right)$ into $L^{2}\left(\mathbb{R}^{n}\right)$ defined by

$$
(\pi(\xi) u)(x)=e^{i x \cdot \xi} u(x), \quad x, \xi \in \mathbb{R}^{n},
$$

for all functions $u$ in $L^{2}\left(\mathbb{R}^{n}\right)$.

PROPOSITION 2.1. Let $\phi$ be any function in $L^{2}\left(\mathbb{R}^{n}\right) \cap L^{\infty}\left(\mathbb{R}^{n}\right)$ such that $\|\phi\|_{2}=1$. Then, for all functions $u$ and $v$ in the Schwartz space $\mathscr{S}$,

$$
(2 \pi)^{-n} \int_{\mathbb{R}^{n}}(u, \pi(\xi) \phi)(\pi(\xi) \phi, v) d \xi=(\bar{\phi} u, \bar{\phi} v),
$$

where $($,$) is the inner product in L^{2}\left(\mathbb{R}^{n}\right)$.

PROOF. Using the Plancherel theorem and the fact that $(\pi(\xi) \phi)^{-}=T_{-\xi} \hat{\phi}$, where

$$
\left(T_{-\xi} f\right)(x)=f(x-\xi), \quad x \in \mathbb{R}^{n},
$$

for any measurable function $f$ on $\mathbb{R}^{n}$, we get

$$
(u, \pi(\xi) \phi)=(\hat{u} * \hat{\psi})(\xi),
$$


and

$$
(\pi(\xi) \phi, v)=\overline{(\hat{v} * \hat{\psi})(\xi)}
$$

for all $\xi$ in $\mathbb{R}^{n}$, where

$$
\psi(x)=\overline{\phi(x)}, \quad x \in \mathbb{R}^{n},
$$

and

$$
(\hat{f} * \hat{\psi})(\xi)=\int_{\mathbb{R}^{n}} \hat{f}(\xi-\eta) \hat{\psi}(\eta) d \eta, \quad \xi \in \mathbb{R}^{n}
$$

for any function $f$ in $L^{2}\left(\mathbb{R}^{n}\right)$. Thus, by (2.3), (2.4), (2.5), the Plancherel theorem and the fact that

$$
(\hat{f} * \hat{g})=(2 \pi)^{n / 2} \tilde{f} \tilde{g}, \quad f, g \in \mathscr{S},
$$

where

$$
\tilde{f}(x)=f(-x), \quad x \in \mathbb{R}^{n}, \quad f \in \mathscr{S},
$$

we get

$$
\int_{\mathbb{R}^{n}}(u, \pi(\xi) \phi)(\pi(\xi) \phi, v) d \xi=(2 \pi)^{n}(\bar{\phi} u, \bar{\phi} v)
$$

and hence (2.2).

The following proposition gives us more information about the constant $(2 \pi)^{-n}$ in formula (2.2).

PROPOSITION 2.2. Let $\phi$ be any function in $L^{2}\left(\mathbb{R}^{n}\right) \cap L^{\infty}\left(\mathbb{R}^{n}\right)$ such that $\|\phi\|_{2}=1$ and let $c_{\phi}$ be the constant defined by

$$
c_{\phi}=\int_{\mathbb{R}^{n}}|(\phi, \pi(\xi) \phi)|^{2} d \xi
$$

Then

$$
c_{\phi}=(2 \pi)^{n}\|\phi\|_{4}^{4},
$$

where \|\|$_{4}$ is the norm in $L^{4}\left(\mathbb{B}^{n}\right)$. 
PROOF. Using the Plancherel theorem, (2.3), (2.5), (2.6) and (2.7), we obtain

$$
\begin{aligned}
\int_{\mathbb{R}^{n}}|(\phi, \pi(\xi) \phi)|^{2} d \xi & =\int_{\mathbb{R}^{n}}|(\hat{\phi} * \hat{\psi})(\xi)|^{2} d \xi \\
& =(2 \pi)^{n} \int_{\mathbb{R}^{n}}|\tilde{\phi}(x) \tilde{\psi}(x)|^{2} d x \\
& =(2 \pi)^{n}\|\phi\|_{4}^{4} .
\end{aligned}
$$

REMARK 2.3. In view of Proposition 2.2, we can rewrite (2.2) as

$$
\frac{1}{c_{\phi}} \int_{\mathbb{R}^{n}}(u, \pi(\xi) \phi)(\pi(\xi) \phi, v) d \xi=\frac{1}{\|\phi\|_{4}^{4}}(\vec{\phi} u, \bar{\phi} v), \quad u, v \in \mathscr{S} .
$$

This reformulation allows us to give an interesting and fruitful comparison of Proposition 2.1 with the resolution of the identity formula used in the paper [3] by He and Wong. To wit, let $G$ be a locally compact and Hausdorff group with a left Haar measure $\mu$. Let $X$ be a separable and complex Hilbert space, the dimension of which is infinite. We denote the inner product and norm in $X$ by $(,)_{X}$ and \|\|$_{X}$ respectively. Let $B(X)$ be the $C^{*}$-algebra of all bounded linear operators from $X$ into $X$. An irreducible unitary representation $\pi: G \rightarrow B(X)$ is said to be square integrable if there exists a non-zero element $\phi$ in $X$ such that

$$
c_{\phi}=\int_{G}\left|(\phi, \pi(g) \phi)_{X}\right|^{2} d \mu(g)<\infty .
$$

We call any non-zero element $\phi$ in $X$ for which (2.9) is valid an admissible wavelet for the representation $\pi: G \rightarrow B(X)$. Using the theory of square integrable representations studied in Grossmann, Morlet and Paul [1, 2] and Holschneider [4], among others, it can be proved that the resolution of the identity formula, that is,

$$
(x, y)_{X}=\frac{1}{c_{\phi}} \int_{G}(x, \pi(g) \phi)_{X}(\pi(g) \phi, y)_{X} d \mu(g), \quad x, y \in X,
$$

is valid. Thus, for the particular unitary representation $\pi: \mathbb{R}^{n} \rightarrow B\left(L^{2}\left(\mathbb{R}^{n}\right)\right)$ studied in this paper, formula (2.2) can be considered as an analogue of the resolution of the identity formula.

The main result in this section is the following theorem.

THEOREM 2.4. Let $\sigma \in L^{\infty}\left(\mathbb{R}^{n}\right)$ and let $\phi$ be any function in $L^{2}\left(\mathbb{R}^{n}\right) \cap L^{\infty}\left(\mathbb{R}^{n}\right)$ such that $\|\phi\|_{2}=1$. If, for any function $u$ in $\mathscr{S}$, we define $\tilde{P}_{\sigma} u$ by

$$
\left(\tilde{P}_{\sigma} u, v\right)=(2 \pi)^{-n} \int_{\mathbb{R}^{n}} \sigma(\xi)(u, \pi(\xi) \phi)(\pi(\xi) \phi, v) d \xi, \quad v \in \mathscr{S},
$$

then

$$
\left(\tilde{P}_{\sigma} u, v\right)=\left(\left(\phi T_{\sigma} \bar{\phi}\right) u, v\right), \quad u, v \in \mathscr{S}
$$


PROOF. Using (2.3), (2.4), (2.5) and the fact that

$$
(f g)^{\widehat{N}}=(2 \pi)^{-n / 2}(\hat{f} * \hat{g}), \quad f, g \in \mathscr{S},
$$

we obtain

$$
\left(\tilde{P}_{\sigma} u, v\right)=\int_{\mathbb{R}^{n}} \sigma(\xi)(\psi u)(\xi) \overline{(\psi v) \mathcal{( \xi )}} d \xi, \quad u, v \in \mathscr{S} .
$$

Thus, by (2.12) and the definition of $T_{\sigma}$ given in Section 1, we get

$$
\left(\tilde{P}_{\sigma} u, v\right)=\left(T_{\sigma}(\psi u), \psi v\right)=\left(\left(\bar{\psi} T_{\sigma} \psi\right) u, v\right)=\left(\left(\phi T_{\sigma} \bar{\phi}\right) u, v\right), \quad u, v \in \mathscr{S},
$$

and the proof is complete.

REMARK 2.5. Had the "admissible wavelet" $\phi$ in (2.10) been replaced by the function $\phi_{0}$ on $\mathbb{R}^{n}$ given by

$$
\phi_{0}(x)=1, \quad x \in \mathbb{R}^{n},
$$

we would have obtained

$$
\left(\tilde{P}_{\sigma} u, v\right)=\left(T_{\sigma} u, v\right), \quad u, v \in \mathscr{S},
$$

that is, $\tilde{P}_{\sigma}$ would have been a "constant coefficient" pseudo-differential operator, or a Fourier multiplier studied in the book $[13]$ or the papers $[14,15]$ by Wong. In view of the fact that the function $\phi$ in the linear operator $\phi T_{\sigma} \bar{\phi}$ is the admissible wavelet in the linear operator $\tilde{P}_{\sigma}$, it is appropriate to call the pseudo-differential operator $\phi T \bar{\phi}: L^{2}\left(\mathbb{B}^{n}\right) \rightarrow L^{2}\left(\mathbb{R}^{n}\right)$ a wavelet multiplier.

\section{Boundedness}

PROPOSITION 3.1. Let $\sigma \in L^{1}\left(\mathbb{R}^{n}\right)$ and let $\phi$ be any function in $L^{2}\left(\mathbb{B}^{n}\right) \cap L^{\infty}\left(\mathbb{R}^{n}\right)$ such that $\|\phi\|_{2}=1$. If, for any function $u$ in $\mathscr{S}$, we define $P_{\sigma} u$ by

$$
P_{\sigma} u=\frac{1}{\|\phi\|_{4}^{4}} \tilde{P}_{\sigma} u,
$$

where $\tilde{P}_{\sigma} u$ is given by (2.10) for all functions $v$ in $\mathscr{S}$, then $P_{\sigma}$ can be extended uniquely to a bounded linear operator from $L^{2}\left(\mathbb{R}^{n}\right)$ into $L^{2}\left(\mathbb{R}^{n}\right)$, again denoted by $P_{\sigma}$, and

$$
\left\|P_{\sigma}\right\|_{*} \leq \frac{1}{c_{\phi}}\|\sigma\|_{1},
$$

where \|\|$_{*}$ is the norm in the $C^{*}$-algebra $B\left(L^{2}\left(\mathbb{R}^{n}\right)\right)$, and \|\|$_{1}$ is the norm in $L^{1}\left(\mathbb{B}^{n}\right)$. 
REMARK 3.2. The proof of Proposition 2.1 in the paper [3] by He and Wong can be used to prove Proposition 3.1. Because of Proposition 3.1 and (2.11), it is natural to define $\phi T_{\sigma} \bar{\phi}: L^{2}\left(\mathbb{R}^{n}\right) \rightarrow L^{2}\left(\mathbb{R}^{n}\right)$, for any function $\sigma$ in $L^{1}\left(\mathbb{R}^{n}\right)$, to be the bounded linear operator $\|\phi\|_{4}^{4} P_{\sigma}: L^{2}\left(\mathbb{R}^{n}\right) \rightarrow L^{2}\left(\mathbb{R}^{n}\right)$. Thus $\phi T_{\sigma} \bar{\phi}$ is a scalar multiple of a variant of a localization operator studied in the paper [3] by He and Wong.

We can now define $\phi T_{\sigma} \bar{\phi}: L^{2}\left(\mathbb{R}^{n}\right) \rightarrow L^{2}\left(\mathbb{R}^{n}\right)$, for any function $\sigma$ in $L^{p}\left(\mathbb{R}^{n}\right)$, $1<p<\infty$. To do this, we use the following theorem which is an analogue of Theorem 3.1 in the paper [3] by He and Wong.

THEOREM 3.3. Let $\sigma \in L^{p}\left(\mathbb{R}^{n}\right), 1<p<\infty$, and let $\phi$ be any function in $L^{2}\left(\mathbb{R}^{n}\right) \cap L^{\infty}\left(\mathbb{R}^{n}\right)$ such that $\|\phi\|_{2}=1$. Then there exists a unique bounded linear operator $P_{\sigma}: L^{2}\left(\mathbb{R}^{n}\right) \rightarrow L^{2}\left(\mathbb{R}^{n}\right)$ such that

$$
P_{\sigma} u=\frac{1}{\|\phi\|_{4}^{4}} \tilde{P}_{\sigma} u, \quad u \in \mathscr{S}
$$

and

$$
\left\|P_{\sigma}\right\|_{*} \leq \frac{\|\phi\|_{\infty}^{\frac{2}{p^{j}}}}{(2 \pi)^{\frac{n}{p}}\|\phi\|_{4}^{4}}\|\sigma\|_{p},
$$

where $\tilde{P}_{\sigma} u$ is given by (2.11) for all functions $u$ and $v$ in $\mathscr{S}$, and all simple functions $\sigma$ on $\mathbb{R}^{n}$ satisfying

$$
\mu\left\{\xi \in \mathbb{R}^{n}: \sigma(\xi) \neq 0\right\}<\infty
$$

where $\mu\{\cdots\}$ is the Lebesgue measure of $\{\cdots\},\|\|_{p}$ is the norm in $L^{p}\left(\mathbb{R}^{n}\right),\|\|_{\infty}$ is the norm in $L^{\infty}\left(\mathbb{R}^{n}\right)$ and $p^{\prime}$ is the conjugate index of $p$.

Proof. By (2.2), (2.10) and the Schwarz inequality, $\tilde{P}_{\sigma}$ can be extended uniquely to a bounded linear operator, again denoted by $\tilde{P}_{\sigma}$, from $L^{2}\left(\mathbb{R}^{n}\right)$ into $L^{2}\left(\mathbb{R}^{n}\right)$ and

$$
\left\|\tilde{P}_{\sigma}\right\|_{*} \leq\|\phi\|_{\infty}^{2}\|\sigma\|_{\infty}, \quad \sigma \in L^{\infty}\left(\mathbb{R}^{n}\right)
$$

Thus, by (2.8), (3.1), (3.2), (3.3) and the Riesz-Thorin theorem in, say, Section IX.4 of the book [7] by Reed and Simon, the proof of Theorem 3.3 is complete.

REMARK 3.4. Theorem 3.3 allows us to define $\phi T_{\sigma} \bar{\phi}: L^{2}\left(\mathbb{R}^{n}\right) \rightarrow L^{2}\left(\mathbb{R}^{n}\right)$, for any function $\sigma$ in $L^{p}\left(\mathbb{R}^{n}\right), 1<p<\infty$, to be the bounded linear operator $\|\phi\|_{4}^{4} P_{\sigma}$ : $L^{2}\left(\mathbb{R}^{n}\right) \rightarrow L^{2}\left(\mathbb{R}^{n}\right)$. 


\section{The Schatten-von Neumann property}

Let $X$ be a separable and complex Hilbert space, the dimension of which is infinite. Let $A: X \rightarrow X$ be a compact operator, and let $A^{*}: X \rightarrow X$ be the adjoint of $A: X \rightarrow X$. Then the linear operator $\left(A^{*} A\right)^{\frac{1}{2}}: X \rightarrow X$ is positive and compact. Let $\left\{\psi_{k}: k=1,2, \ldots\right\}$ be an orthonormal basis for $X$ consisting of eigenvectors of $\left(A^{*} A\right)^{\frac{1}{2}}: X \rightarrow X$, and let $s_{k}(A)$ be the eigenvalue corresponding to the eigenvector $\psi_{k}, k=1,2, \ldots$ Then the compact operator $A: X \rightarrow X$ is said to be in the Schatten-von Neumann class $S_{p}, 1 \leq p<\infty$, if

$$
\sum_{k=1}^{\infty} s_{k}(A)^{p}<\infty
$$

It can be shown that $S_{p}, 1 \leq p<\infty$, is a Banach space in which the norm \|\|$_{S_{p}}$ is given by

$$
\|A\|_{s_{p}}=\left\{\sum_{k=1}^{\infty} s_{k}(A)^{p}\right\}^{\frac{1}{p}}, \quad A \in S_{p} .
$$

The Schatten-von Neumann class $S_{\infty}$, by convention, is the $C^{*}$ - algebra $B(X)$ of all bounded linear operators from $X$ into $X$.

A detailed study of the properties of the Schatten-von Neumann class $S_{p}, 1 \leq p \leq$ $\infty$, can be found in Reed and Simon [8], Simon [9] and Zhu [16].

The following proposition is an analogue of Proposition 5.1 in the paper [3] by $\mathrm{He}$ and Wong.

Proposition 4.1. Let $\sigma \in L^{1}\left(\mathbb{R}^{n}\right)$ and let $\phi$ be any function in $L^{2}\left(\mathbb{R}^{n}\right) \cap L^{\infty}\left(\mathbb{R}^{n}\right)$ such that $\|\phi\|_{2}=1$. Then the pseudo-differential operator $\phi T_{\sigma} \bar{\phi}: L^{2}\left(\mathbb{R}^{n}\right) \rightarrow L^{2}\left(\mathbb{R}^{n}\right)$ defined in Section 3 is in $S_{1}$ and

$$
\left\|\phi T_{\sigma} \bar{\phi}\right\|_{S_{1}} \leq \frac{4}{(2 \pi)^{n}}\|\sigma\|_{1} .
$$

Remark 4.2. The proof of Proposition 4.1 is exactly the same as the proof of Proposition 5.1 in He and Wong [3] and hence is omitted.

The main result in this section is the following theorem. It is an immediate consequence of (3.2), (4.1) and the theory of complex interpolation given in Section 2.2 of the book [16] by Zhu.

THEOREM 4.3. Let $\sigma \in L^{p}\left(\mathbb{R}^{n}\right), 1 \leq p \leq \infty$, and let $\phi$ be any function in $L^{2}\left(\mathbb{R}^{n}\right) \cap L^{\infty}\left(\mathbb{R}^{n}\right)$ such that $\|\phi\|_{2}=1$. Then the pseudo-differential operator $\phi T_{\sigma} \bar{\phi}$ : 
$L^{2}\left(\mathbb{R}^{n}\right) \rightarrow L^{2}\left(\mathbb{R}^{n}\right)$, defined in Sections 2 and 3 , is in $S_{p}$ and

$$
\left\|\phi T_{\sigma} \bar{\phi}\right\|_{S_{p}} \leq \frac{4^{1 / p}\|\phi\|_{\infty}^{2 / p^{\prime}}}{(2 \pi)^{\frac{n}{p}}\|\phi\|_{4}^{4}}\|\sigma\|_{p} .
$$

\section{The Landau, Pollak and Slepian opearator}

Let $\Omega$ and $T$ be positive numbers. Then we define the linear operators $P_{\Omega}$ : $L^{2}(\mathbb{R}) \rightarrow L^{2}(\mathbb{R})$ and $Q_{T}: L^{2}(\mathbb{R}) \rightarrow L^{2}(\mathbb{R})$ by

$$
\left(P_{\Omega} f\right)(\xi)= \begin{cases}\hat{f}(\xi), & |\xi| \leq \Omega, \\ 0, & |\xi|>\Omega,\end{cases}
$$

and

$$
\left(Q_{T} f\right)(x)= \begin{cases}f(x), & |x| \leq T, \\ 0, & |x|>T,\end{cases}
$$

for all functions $f$ in $L^{2}(\mathbb{R})$. Then we can see easily that $P_{\Omega}: L^{2}(\mathbb{R}) \rightarrow L^{2}(\mathbb{R})$ and $Q_{T}: L^{2}(\mathbb{R}) \rightarrow L^{2}(\mathbb{R})$ are self-adjoint projections. In signal analysis, a signal is a function $f$ in $L^{2}(\mathbb{R})$. Thus, for any function $f$ in $L^{2}(\mathbb{R})$, the function $Q_{T} P_{\Omega} f$ can be considered to be a time and band-limited signal. Therefore it is of interest to compare the energy $\left\|Q_{T} P_{\Omega} f\right\|_{2}^{2}$ of the time and band-limited signal $Q_{T} P_{\Omega} f$ with the energy $\|f\|_{2}^{2}$ of the original signal $f$. Using the fact that $P_{\Omega}$ and $Q_{r}$ are self-adjoint, and the fact that $Q_{T}$ is a projection, we get

$$
\begin{aligned}
\sup & \left\{\frac{\left\|Q_{T} P_{\Omega} f\right\|_{2}^{2}}{\|f\|_{2}^{2}}: f \in L^{2}(\mathbb{R}),\|f\|_{2} \neq 0\right\} \\
& =\sup \left\{\frac{\left(Q_{T} P_{\Omega} f, Q_{T} P_{\Omega} f\right)}{\|f\|_{2}^{2}}: f \in L^{2}(\mathbb{R}),\|f\|_{2} \neq 0\right\} \\
& =\sup \left\{\frac{\left(P_{\Omega} Q_{T} P_{\Omega} f, f\right)}{\|f\|_{2}}: f \in L^{2}(\mathbb{R}),\|f\|_{2}^{2} \neq 0\right\} \\
& =\sup \left\{\left(P_{\Omega} Q_{T} P_{\Omega} f, f\right): f \in L^{2}(\mathbb{R}),\|f\|_{2}=1\right\} .
\end{aligned}
$$

Since $P_{\Omega} Q_{T} P_{\Omega}: L^{2}(\mathbb{R}) \rightarrow L^{2}(\mathbb{R})$ is self-adjoint, it follows from (5.3) that

$$
\sup \left\{\frac{\left\|Q_{T} P_{\Omega} f\right\|_{2}^{2}}{\|f\|_{2}^{2}}: f \in L^{2}(\mathbb{R}),\|f\|_{2} \neq 0\right\}=\left\|P_{\Omega} Q_{T} P_{\Omega}\right\|_{*} .
$$

The bounded linear operator $P_{\Omega} Q_{T} P_{\Omega}: L^{2}(\mathbb{R}) \rightarrow L^{2}(\mathbb{R})$ arising in the study of time and band-limited signals is called the Landau, Pollak and Slepian operator. See 
the fundamental papers $[5,6]$ by Landau and Pollak, $[10,11]$ by Slepian and $[12]$ by Slepian and Pollak in this connection.

Let $\phi$ be the function on $\mathbb{R}$ defined by

$$
\phi(x)= \begin{cases}\frac{1}{\sqrt{2 \Omega}}, & |x| \leq \Omega, \\ 0, & |x|>\Omega .\end{cases}
$$

Then $\phi \in L^{2}(\mathbb{R}) \cap L^{\infty}(\mathbb{R})$ and $\|\phi\|_{2}=1$. Let $\sigma$ be the characteristic function on $[-T, T]$, that is,

$$
\sigma(\xi)= \begin{cases}1, & |\xi| \leq T \\ 0, & |\xi|>T .\end{cases}
$$

Then, by Theorem 2.4 ,

$$
\left(\left(\phi T_{\sigma} \phi\right) u, v\right)=(2 \pi)^{-1} \int_{-\infty}^{\infty} \sigma(\xi)(u, \pi(\xi) \phi)(\pi(\xi) \phi, v) d \xi, \quad u, v \in \mathscr{S}
$$

By (5.1), (5.4) and the Fourier inversion formula,

$$
\begin{aligned}
(u, \pi(\xi) \phi) & =\frac{1}{\sqrt{2 \Omega}} \int_{-\Omega}^{\Omega} e^{-i x \xi} u(x) d x \\
& =\sqrt{\frac{\pi}{\Omega}} \frac{1}{\sqrt{2 \pi}} \int_{-\infty}^{\infty} e^{-i x \xi}\left(P_{\Omega} \check{u}\right)(x) d x \\
& =\sqrt{\frac{\pi}{\Omega}}\left(P_{\Omega} \check{u}\right)(-\xi), \quad u \in L^{2}(\mathbb{R}),
\end{aligned}
$$

for almost all $\xi$ in $\mathbb{R}$, where $\check{u}$ is the inverse Fourier transform of $u$. Hence, by (5.5), (5.6), (5.7) and the Plancherel theorem, we get

$$
\begin{aligned}
\left(\left(\phi T_{\sigma} \phi\right) u, v\right) & =\frac{1}{2 \Omega} \int_{-\infty}^{\infty} \sigma(\xi)\left(P_{\Omega} \check{u}\right)(\xi) \overline{\left(P_{\Omega} \check{v}\right)(\xi)} d \xi \\
& =\frac{1}{2 \Omega}\left(P_{\Omega} Q_{T} P_{\Omega} \check{u}, \check{v}\right) \\
& =\frac{1}{2 \Omega}\left(\mathscr{F} P_{\Omega} Q_{T} P_{\Omega} \mathscr{F}^{-1} u, v\right), \quad u, v \in L^{2}(\mathbb{B}) .
\end{aligned}
$$

So the wavelet multiplier $\phi T_{\sigma} \phi$ is unitarily equivalent to a scalar multiple of the Landau, Pollak and Slepian operator, that is, $P_{\Omega} Q_{r} P_{\Omega} / 2 \Omega$. 


\section{Acknowledgment}

This research has been partially supported by the Natural Sciences and Engineering Research Council of Canada under Grant No. A8562.

\section{References}

[1] A. Grossmann, J. Morlet and T. Paul, "Transforms associated to square integrable group representations I: General results", J. Math. Phys. 26 (1985) 2473-2479.

[2] A. Grossmann, J. Morlet and T. Paul, "Transforms associated to square integrable group representations II: Examples", Ann. Inst. Henri Poincarè 45 (1986) 293-309.

[3] Z. He and M. W. Wong, "Localization operators associated to square integrable group representations", Panamer. Math. J. 6 (1996) 93-104.

[4] M. Holschneider, Wavelets: An Analysis Tool (Oxford University Press, 1995).

[5] H. J. Landau and H. O. Pollak, "Prolate spheroidal wave functions, Fourier analysis and uncertainty, II", Bell Syst. Techn. J. 40 (1961) 65-84.

[6] H. J.Landau and H. O. Pollak, "Prolate spheroidal wave functions, Fourier analysis and uncertainty, III", Bell Syst. Techn. J. 41 (1962) 1295-1336.

[7] M. Reed and B. Simon, Methods of Mathematical Physics II: Fourier Analysis, Self-Adjointness (Academic Press, 1975).

[8] M. Reed and B. Simon, Methods of Mathematical Physics I: Functional Analysis (Academic Press, 1980).

[9] B. Simon, Trace Ideals and their Applications (Cambridge University Press, 1979).

[10] D. Slepian, "On bandwidth", Proc. IEEE 64 (1976) 292-300.

[11] D. Slepian, "Some comments on Fourier analysis, uncertainty and modeling", SIAM Rev 25 (1983) 379-393.

[12] D. Slepian and H. O. Pollak, "Prolate spheroidal wave functions, Fourier analysis and uncertainty, I", Bell Syst. Tech. 40 (1961) 43-64.

[13] M. W. Wong, An Introduction to Pseudo-Differential Operators (World Scientific, 1991).

[14] M. W. Wong, "Spectral theory of pseudo-differential operators", Adv. Appl. Math. 15 (1994) 437-451.

[15] M. W. Wong, “Norms of Fourier Multipliers”, Bull. Inst. Math. Acad. Sinica 23 (1995) 329-334.

[16] K. Zhu, Operator Theory in Function Spaces (Marcel Dekker, 1990). 